\title{
RESOLVING THE MERGING PLANCK CLUSTER PLCK G147.3-16.6 WITH GISMO
}

\author{
T. Mroczkowski ${ }^{1,8}$, A. Kovács ${ }^{2,3}$, E. Bulbul ${ }^{4}$, J. Staguhn ${ }^{5,6}$, D. J. Benford ${ }^{5}$, T. E. Clarke ${ }^{1}$, \\ R. J. VAN WEeren ${ }^{4}$, H. T. INTEMA ${ }^{7}$, AND S. RANDALl ${ }^{4}$ \\ ${ }^{1}$ U.S. Naval Research Laboratory, 4555 Overlook Avenue SW, Washington, D.C. 20375, USA; anthony.mroczkowski.ctr@nrl.navy.mil \\ ${ }^{2}$ California Institute of Technology 301-17, 1200 E California Boulevard, Pasadena, CA 91125, USA \\ ${ }^{3}$ Institute for Astrophysics, University of Minnesota, 116 Church Street SE, Minneapolis, MN 55455, USA \\ ${ }^{4}$ Harvard-Smithsonian Center for Astrophysics, 60 Garden Street, Cambridge, MA 02138, USA \\ ${ }^{5}$ Observational Cosmology Lab., Code 665, NASA at Goddard Space Flight Center, Greenbelt, MD 20771, USA \\ ${ }^{6}$ Department of Physics and Astronomy, Johns Hopkins University, Baltimore, MD, 21218, USA \\ ${ }^{7}$ National Radio Astronomy Observatory, P.O. Box O, 1003 Lopezville Road, Socorro, NM 87801-0387, USA \\ Received 2015 January 20; accepted 2015 June 9; published 2015 July 14
}

\begin{abstract}
The Planck satellite has recently completed an all-sky galaxy cluster survey exploiting the thermal SunyaevZel'dovich (SZ) effect to locate some of the most massive systems observable. With a median redshift of $\langle z\rangle=0.22$, the clusters found by Planck at $z>0.3$ are proving to be exceptionally massive and/or disturbed systems. One notable Planck discovery at $z=0.645$, PLCK G147.3-16.6, has an elongated core and hosts a radio halo, indicating it is likely in the process of merging. We present a $16^{\prime \prime} .5$ resolution SZ observation of this high- $z$ merger using the Goddard-IRAM Superconducting 2-Millimeter Observer, and compare it to X-ray follow-up observations with XMM-Newton. We find the SZ pressure substructure is offset from the core components seen in $\mathrm{X}$-ray. We interpret this as possible line of sight temperature or density substructure due to the on-going merger.
\end{abstract}

Key words: cosmology: observations - galaxies: clusters: general - galaxies: clusters: individual

(PLCK G147.3-16.6) - galaxies: clusters: intracluster medium - X-rays: galaxies: clusters

\section{INTRODUCTION}

Forming from the largest fluctuations in the primordial matter power spectrum, galaxy clusters are among the most massive gravitationally bound objects. Therefore, the distribution of clusters as a function of mass and redshift provides sensitive cosmological probes. Surveys spanning the electromagnetic spectrum are planned or underway to catalog clusters across their formation history. Recent efforts exploiting the redshift-independent surface brightness of the SunyaevZel'dovich effect (SZ; Sunyaev and Zel'dovich 1972) in particular have detected $\sim 1000$ previously unknown clusters (see Fowler et al. 2010; Carlstrom et al. 2011; Planck Collaboration I 2011; Planck Collaboration VIII 2011; Hasselfield et al. 2013; Planck Collaboration XXIX 2014; Bleem et al. 2015).

The Planck satellite has completed the first all-sky cluster survey since ROSAT (see, e.g., Romer et al. 1994; Voges et al. 1999; Böhringer et al. 2000). Planck, however, is not well-suited for the discovery of high- $z$ systems, whose arcminute-scale SZ signals are heavily diluted inside Planck's 7!3-9!7 beams at the detecting 2 and $3 \mathrm{~mm}$ bands. As such, Planck detects only the most prominent, rare systems at high- $z$. The Planck XMM-Newton cluster validation program (Planck Collaboration IV 2013) used the 15.5 month nominal survey data to identify likely cluster candidates and understand Planck's selection function. It suggests that the high-z detections are likely dynamically disturbed massive systems, which are far from being virialized and, on average, less X-ray luminous than X-ray selected clusters of the same mass.

Here we report high-significance $16^{\prime \prime} .5$ resolution SZ observations of a disturbed cluster from the final Planck $X M M-N e w t o n$ cluster validation program, imaging it with nearly $20 \times$ better resolution than its original unresolved

\footnotetext{
${ }^{8}$ National Research Council Fellow, National Academy of Sciences.
}

detection. These new data, from the Goddard-IRAM 2-Millimeter Observer (GISMO; Staguhn et al. 2006) on the $30 \mathrm{~m}$ Institut de Radioastronomie Millimétrique (IRAM) Telescope ${ }^{9}$ on Pico Veleta, Spain, reveal a complex pressure substructure in this merging system and underscore the power of subarcminute SZ follow-up.

We summarize the known cluster properties in Section 2, discuss the new observations in Section 3, and present the results of our analysis in Section 4 . We adopt a $\Lambda \mathrm{CDM}$ cosmology with $\Omega_{M}=0.3, \quad \Omega_{\Lambda}=0.7, \quad$ and $H_{0}=70 \mathrm{~km} \mathrm{~s}^{-1} \mathrm{Mpc}^{-1}$ throughout this paper. At the redshift of PLCK G147.3-16.6 $(z=0.645$; Planck Collaboration IV 2013), $1^{\prime \prime}$ corresponds to $6.9 \mathrm{kpc}$.

\section{PLCK G147.3-16.6}

PLCK G147.3-16.6 is a massive cluster at $z=0.645$, discovered at a signal-to-noise ratio $(\mathrm{S} / \mathrm{N})=4.41$ in the nominal 15.5 month Planck mission. X-ray follow-up observations in the Planck XMM-Newton validation program (Planck Collaboration IV 2013) reveal an extended, double core morphology, while optical observations with Gemini show no cD galaxy dominating the cluster field. More recently, $610 \mathrm{MHz}$ observations with the Giant Metrewave Radio Telescope (GMRT) found that PLCK G147.316.6 hosts a $0.9 \mathrm{Mpc}$ radio halo (van Weeren et al. 2014), placing it among the highest redshift radio halos known. The (re-)acceleration processes that create radio halos are thought to occur predominantly in mergers during and after the first core passage (Feretti et al. 2012; Brunetti \& Jones 2014), although at least one relaxed, cool-core cluster is known to host a radio halo (Bonafede et al. 2014). The disturbed X-ray

\footnotetext{
This work is based on observations carried out with the $30 \mathrm{~m}$ IRAM Telescope. IRAM is supported by INSU/CNRS (France), MPG (Germany), and IGN (Spain).
} 
Table 1

Observations

\begin{tabular}{|c|c|c|c|c|c|}
\hline Observatory & Date & Project Code & \multicolumn{2}{|c|}{ Pointing (J2000) } & $\begin{array}{c}\text { Clean Exposure Time } \\
\qquad(\mathrm{ks})\end{array}$ \\
\hline GISMO & 2014 Apr 7-9 & $235-13$ & $02: 56: 20.0$ & $+40: 17: 21.0$ & 16.9 \\
\hline XMM-Newton & 2012 Aug 27 & $0693 \overline{6} 61601$ & $02: 56: 23.8$ & $+40: 17: 28.0$ & $41.7 / 42.1 / 32.9^{\mathrm{a}}$ \\
\hline XMM-Newton & 2011 Aug 22 & 0679181301 & $02: 56: 25.3$ & $+40: 17: 18.7$ & $15.4 / 15.7 / 8.6^{\mathrm{a}}$ \\
\hline
\end{tabular}

Notes. Aim points and unflagged exposure times for the observations of PLCK G147.3-16.6 included here.

a Times for the MOS1, MOS2, and PN detectors of XMM-Newton EPIC, respectively.
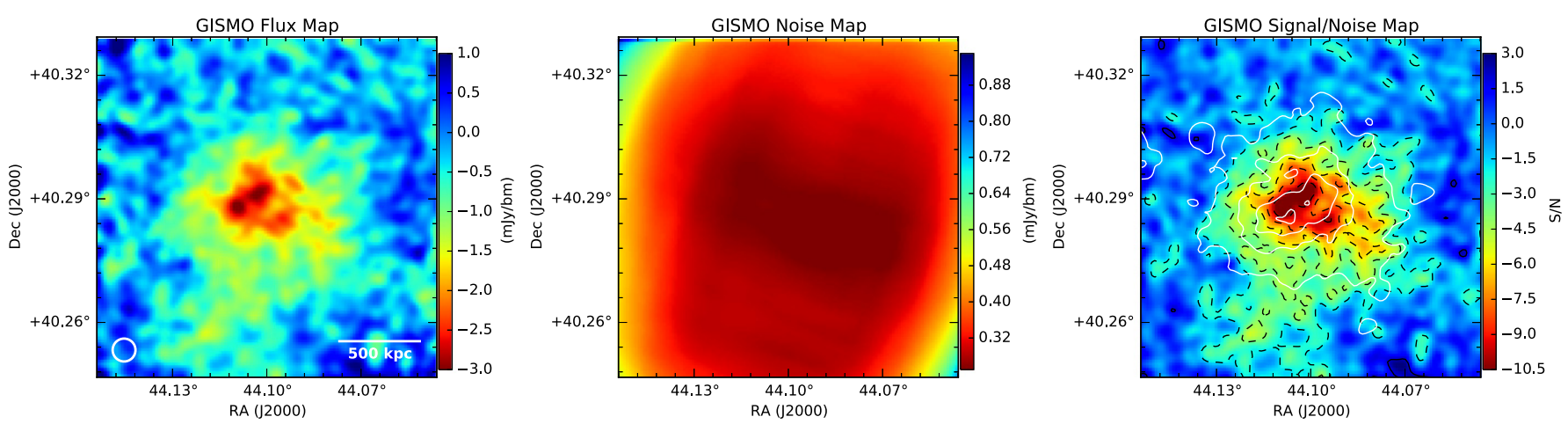

Figure 1. Left: deconvolved GISMO flux map, smoothed by a $12^{\prime \prime}$ Gaussian to a resolution of 19 ". 2 (depicted in the lower left corner), showing the SZ decrement $(\mathrm{mJy} / \mathrm{bm})$ toward PLCK G147.3-16.6. Middle: GISMO noise map with nearly uniform coverage in the region where the cluster is best detected. Right: signal-to-noise ratio $(\mathrm{S} / \mathrm{N}$ ) map (the ratio of left and middle images), with contours at $\mathrm{S} / \mathrm{N}=[4,2,-2,-4,-6,-8,-10]$ overlaid in black. X-ray contours from Figure 3 (left panel) are overlaid in white, starting at $3 \sigma$ and spaced at $3 \sigma$ intervals.

morphology and lack of a dominant $\mathrm{cD}$ galaxy indicate that PLCK G147.3-16.6 belongs to the traditional category of merging clusters hosting radio halos.

\section{OBSERVATIONS}

In this section we describe the resolved GISMO SZ observations and new XMM-Newton data presented in this work. Table 1 summarizes the observations presented here, including those from the GMRT reported in van Weeren et al. (2014) and the previous XMM-Newton observations in Planck Collaboration IV (2013).

\subsection{GISMO}

We observed PLCK G147.3-16.6 for a total of $4.7 \mathrm{hr}$ $(16.9 \mathrm{ks})$ in 2014 April using GISMO, an $8 \times 16$ element array of transition edge sensors. From the $30 \mathrm{~m}$ IRAM Telescope, GISMO provides a $1 ! 8 \times 3 ! 8$ instantaneous field of view with $16^{\prime \prime} .5$ resolution at $150 \mathrm{GHz}(2 \mathrm{~mm})$. The GISMO data were reduced with $C R U S H^{10}$ (ver. 2.22-1; Kovács 2008), which was optimized to recover extended, diffuse signals from the atmosphere-dominated bolometer data.

We estimate a total calibration uncertainty $\sim 7 \%-9 \%$ due to the imperfect knowledge of the line of sight opacities, based on repeated observations of Mars and Uranus (see Staguhn et al. 2014, for a detailed description of the absolute calibration).

We deconvolved the resulting image with the measured point-source response of the reduction process; i.e., we divide the Fourier transform of the map by the 2D transfer function,

\footnotetext{
${ }^{10}$ http://www.submm.caltech.edu/ sharc/crush
}

and back-transform. The resulting deconvolved image is shown in Figure 1 (left).

The cluster field was observed using a combination of $3^{\prime}-$ $5^{\prime}$ alt-azimuthal Lissajous patterns, yielding a median noise $\approx 0.3 \mathrm{mJy} \mathrm{bm}^{-1}$ within the central $4^{\prime}$ diameter, and coverage extending to approximately $6 ! 3 \times 8.3$ area overall.

The noise in each map pixel was propagated from noise measured in the residual detector timestreams. Non-white (covariant) features, such as residual $1 / f$, is spatially invariant, and hence fully captured by an appropriate noise re-scaling, which we determined by the ratio of measured-to-expected deviation, $\left\langle\left(S_{i, j} / \sigma_{i, j}\right)^{2}\right\rangle^{1 / 2}$, outside of the approximate cluster center (at $r>1 ! 5$ ). The resulting noise map, shown in Figure 1 (middle), provides a fair measure of the true map noise for GISMO, with no apparent transient noise. We find the peak SZ decrement at $>10 \sigma$ significance, and the overall detection is significant at $>3 \sigma$ in every beam within the central $\gtrsim 2^{\prime}$ of the map.

The two-dimensional transfer function of our GISMO data, shown in Figure 2, was obtained by inserting a faint point-like test source into jackknife realizations, which are reduced the same way as the cluster. Thus, we ensure that the test data set has the same noise properties as the actual data set, and therefore the test source undergoes the same filtering steps as our cluster, even with adaptive pipeline steps such as noise whitening. We averaged the response over 100 jackknife realizations to suppress the low-level sky-noise present in the individual realizations. The transfer function, obtained as the ratio of the observed 2D spatial spectrum of the response to the underlying spectrum of the test source, characterizes the pipeline's response to arbirtrary structures. 

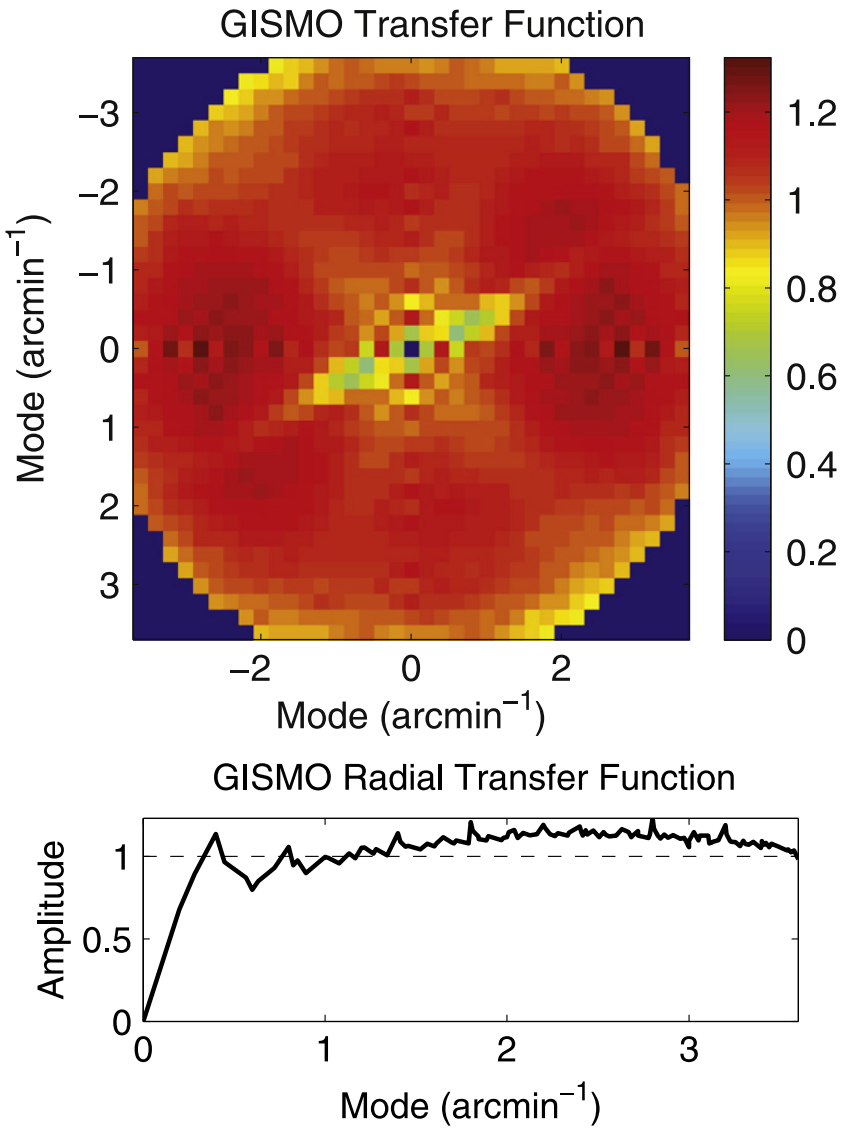

Figure 2. Upper: 2D transfer function used for deconvolution of the PLCK G147.3-16.6 GISMO observation. Note: the corner values at radii $>3.2$ arcmin $^{-1}$ are indeterminate. Lower: radial profile of the above transfer function.

Our transfer function is not circularly symmetric due to common mode subtraction along various correlated detector groups. Because CRUSH normalizes maps to preserve pointsource peak fluxes (i.e., to keep the point source weighted mean response unity by definition), the response in the raw GISMO map diminishes gradually from above unity at the short spatial scales $\left(20^{\prime \prime} ; 3 \operatorname{arcmin}^{-1}\right)$, to $<1$ at scales $>2^{\prime}$, which is comparable to the instantaneous field of view. The azimuthally averaged transfer function is also shown in Figure 2 (lower panel).

The raw map deconvolved by the transfer function provides an accurate representation of the underlying $2 \mathrm{~mm}$ flux distribution of PLCK G147.3-16.6 up to the 5' scales shown. The reduction and deconvolution algorithm was tested on both a simulated point source and a simulated cluster model, and it accurately reproduced the expected fluxes and profiles for both (see Figure 4). The zero level of the deconvolved map is estimated using the mean flux level outside the cluster decrement (at radii $R>1.5$; see Section 4).

\subsection{XMM-Newton}

The discovery of PLCK G147.3-16.6 was confirmed through a $16.9 \mathrm{ks}$ X-ray observation with the XMM-Newton European Photon Imaging Camera (EPIC) in 2011 August, as part of the Planck XMM-Newton validation program (Planck Collaboration IV 2013). An additional $43.9 \mathrm{ks} X M M-N e w t o n$ observation was obtained in 2012 August. We calibrated both data sets using the
Science Analysis System (SAS, ver. 13.5.0) and the most recent calibration files as of 2014 July. The calibrated, cleaned event files discard periods of high intensity due to background particle flares. Effective exposure times and other observation details are summarized in Table 1.

The images and spectra were cleaned of point sources. CCD4 of the MOS1 detector was operating in an anomalous state during observation 0679181301, and therefore excluded from further analysis. The merged, exposure-corrected X-ray surface brightness $\left(S_{\mathrm{X}}\right)$ image in Figure 3 (left) shows that the cluster has a disturbed morphology, with an elongated core angled $\approx 30^{\circ}$ counterclockwise from E-W.

We extracted spectra for the temperature analysis using the SAS tools mos-spectra and pn-spectra. Extracted MOS1 +MOS2 spectra were co-added, and the MOS and PN spectra were jointly fit using xspec using cstat statistics. Each region contained >2000 background-subtracted (i.e., source) counts. With the column density of hydrogen held fixed at the Galactic value, we fit the APEC plasma model to find the temperature of each region, marginalizing over abundance. Further details of the data reduction and analysis such as the treatments of the local, cosmic, and particle backgrounds are discussed in Bulbulx et al. (2012).

We used the XMM-Newton spectroscopic data to produce a temperature $\left(k_{\mathrm{B}} T_{\mathrm{e}}\right)$ map of the cluster using the contour binning algorithm of Sanders (2006), contbin, which selects regions of similar $S_{\mathrm{X}}$ above a user-specified $\mathrm{S} / \mathrm{N}$ threshold. We used $\mathrm{S} / \mathrm{N}>30 y i e l d i n g>2000$ source counts per region. The resulting temperature map is shown in Figure 3 (middle panel), with GISMO contours overlaid for comparison. A pseudo-pressure map $\left(k_{\mathrm{B}} T_{\mathrm{e}} \times \sqrt{S_{\mathrm{X}}}\right)$ is shown in Figure 3 (right).

\section{RESULTS}

In this section we compare the surface brightness of the GISMO SZ map with the model fit to the Planck data and to the properties inferred from the XMM-Newton observations. The thermal SZ effect traces the line-of-sight integral of thermal electron pressure $P_{\mathrm{e}}$. Its surface brightness is proportional to the Compton $y$ parameter,

$$
y \equiv \frac{\sigma_{\mathrm{T}}}{m_{\mathrm{e}} c^{2}} \int n_{\mathrm{e}} k_{\mathrm{B}} T_{\mathrm{e}} d \ell=\frac{\sigma_{\mathrm{T}}}{m_{\mathrm{e}} c^{2}} \int P_{\mathrm{e}} d \ell
$$

where $\sigma_{\mathrm{T}}$ is the Thomson cross-section, $k_{\mathrm{B}}$ is the Boltzmann constant, $m_{\mathrm{e}} c^{2}$ is the electron rest energy, $n_{\mathrm{e}}$ is the electron number density, $k_{\mathrm{B}} T_{\mathrm{e}}$ is the electron temperature, $\ell$ is the line of sight path through the cluster, and $P_{\mathrm{e}}=n_{\mathrm{e}} k_{\mathrm{B}} T_{\mathrm{e}}$.

Planck Collaboration IV (2013) report a spherically integrated Compton $Y_{500, \mathrm{sph}}=(5.2 \pm 1.7) \times 10^{-4} \mathrm{arcmin}^{2}$, where

$$
Y_{500, \mathrm{sph}} \equiv \frac{\sigma_{\mathrm{T}}}{m_{\mathrm{e}} c^{2} D_{\mathrm{A}}^{2}} \int_{0}^{R_{500}} P_{\mathrm{e}}(r) 4 \pi r^{2} d r .
$$

Here $D_{\mathrm{A}}$ is the angular diameter distance to the cluster, and $R_{500}$, which for this cluster $=1042 \mathrm{kpc}(2 ! 5$ on the sky), is the radius within which the average density is $500 \times$ greater than the critical density of the Universe at that redshift. Planck Collaboration IV (2013) assume the spherically symmetric "Universal pressure profile" (UPP) of Arnaud et al. (2010) for their model fit, shown in blue on Figure 4. For simplicity, we 

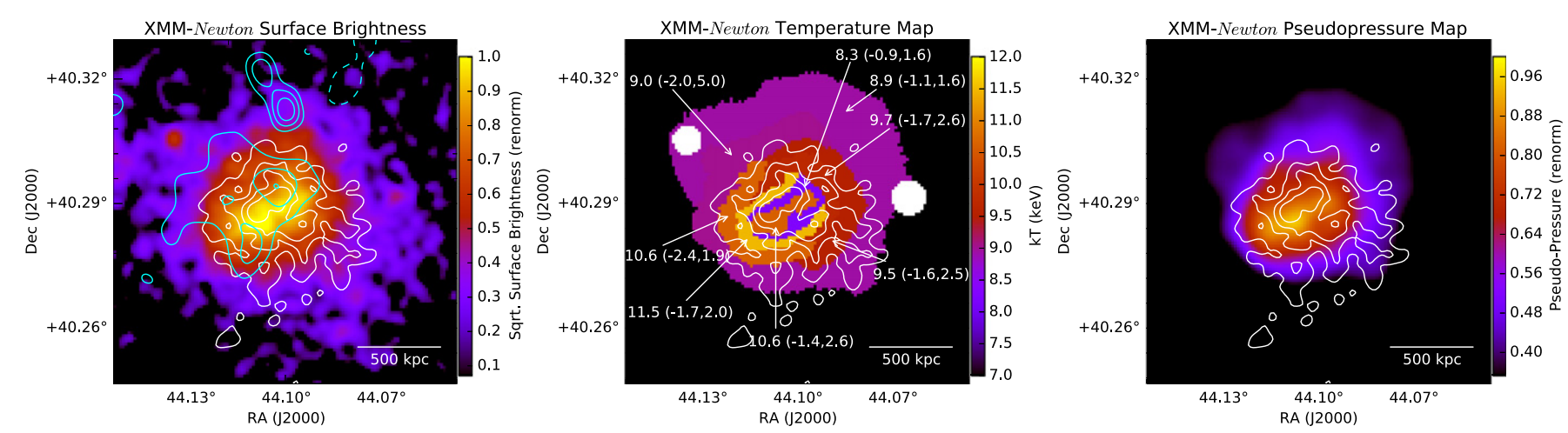

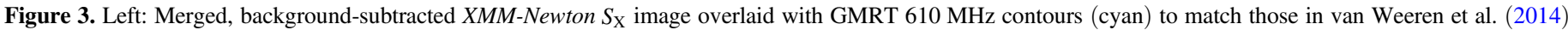

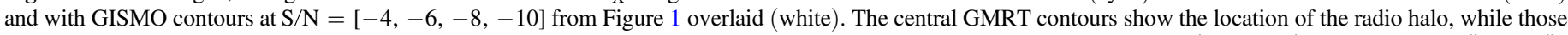

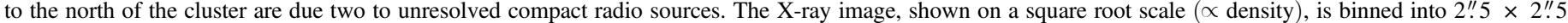

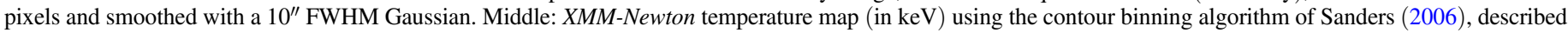

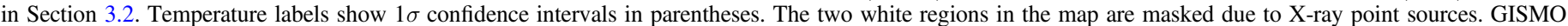

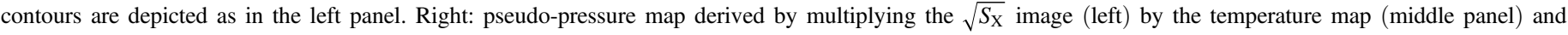
smoothing to the resolution of GISMO.

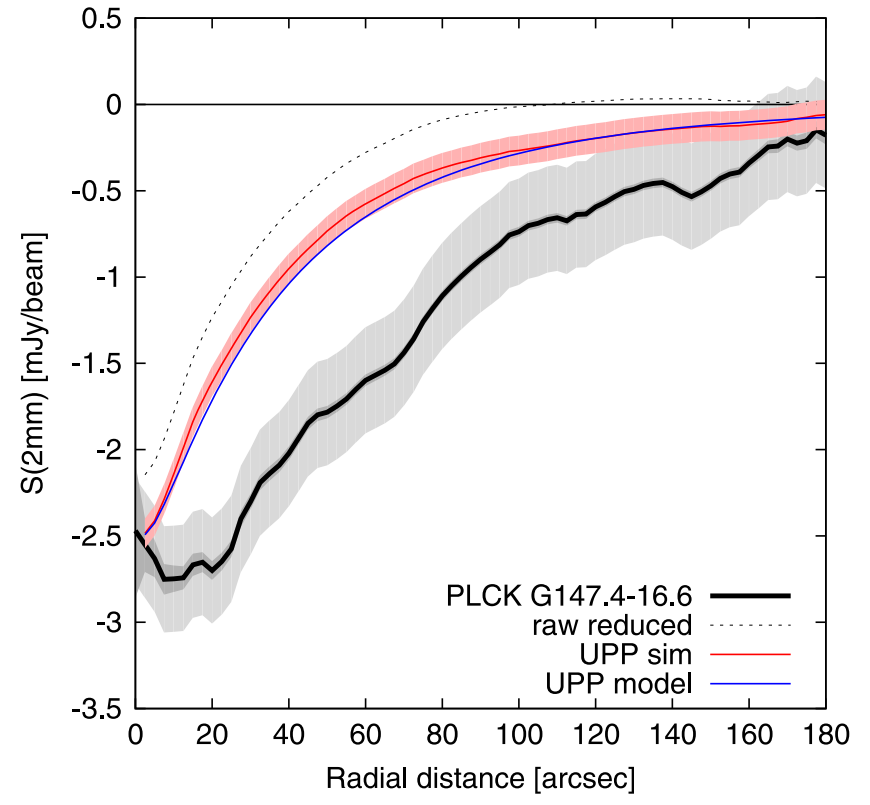

Figure 4. SZ surface brightness profiles from the raw (dotted) and deconvolved (solid black) GISMO maps, with measurement uncertainties (dark gray) and systematic zero-level estimation uncertainty (light gray) ranges, compared with that computed from the median UPP (blue) fit to the Planck data (Planck Collaboration IV 2013). We also show the profile we recover (red with $2 \sigma$ uncertainties) when we insert the UPP into the jackknifed GISMO timestreams and analyze it the same way as the actual cluster observation.

plotted only the median UPP fit to the Planck data, noting the error bars on the SZ surface brightness profile are $\approx 13 \%$ at $R_{500}$, and $\approx 7 \%$ at the peak.

The source of the discrepancy between the measured and modeled profiles is unknown, but may be an indication that the UPP is a poor fit to this disturbed cluster. We note that the UPP as fit by the Planck is treated as a matched filter function of a single parameter, namely the mass within $R_{500}$ as computed using the scalings reported in Arnaud et al. (2010). Furthermore, the Planck measurement of this cluster is entirely unresolved within $R_{500}$, so the profile shown is an interpolation of the UPP that results in the integrated signal measurement by Planck on scales $\gg R_{500}$.
The GISMO S/N map shows broad qualitative agreement with the X-ray imaging (Figure 3, left). In the core, however, we find the SZ and X-ray peaks are offset. An attempt to realign the peaks would bring the agreement on arcminute scales into tension.

The observed peak offsets between the GISMO and XMMNewton maps is not expected to be due to pointing errors. $X M M-N e w t o n$ imaging is precise to the subarcsecond level, and the positions of bright X-ray point sources agree with the locations of bright WISE counterparts. The GISMO pointing model is checked against 466 measurements of bright point sources, observed hourly through several days. All are within an rms deviation $<4$ ". 5 . The GISMO observations combine 32 observation blocks, each bracketed by independent pointings, thus the statistical $1 \sigma$ pointing error of the composite map is estimated at $0 "$. 8 . We also note that the positions of dozens of bright compact sources observed by GISMO observations during the same observation period are reproduced within the expected accuracy. We therefore consider the discrepancy to be of astrophysical nature.

We do not expect to detect any contamination by the Cosmic Infrared Background (CIB). The unresolved part of the CIB is removed by flux zeroing outside of the cluster, so our fluxes are effectively referenced against the mean CIB level. Resolved CIB sources may be present in the map, but are unlikely to be detectable. A deep-field study with GISMO by Staguhn et al. (2014) finds no sources brighter than $\sim 1 \mathrm{mJy}$ ( $>3 \sigma$ in our map) in a similar area to our field, and put the $2 \mathrm{~mm}$ confusion noise at $\lesssim 50 \mu \mathrm{Jy}$, i.e., several times below the $\mathrm{rms}$ in the observations presented here. The combined $\mathrm{CMB}+\mathrm{CIB}$ at scales from $1^{\prime}$ to $4^{\prime}$ has also been measured at $150 \mathrm{GHz}$ by the SPT to be $\lesssim 100 \mu \mathrm{K}$ (George et al. 2015), corresponding to a signal $\lesssim 407 \mu \mathrm{Jy} \mathrm{bm}^{-1}$ in the GISMO data, comparable to the noise level in our map. We also note that recent studies by Sayers et al. (2013) and Gralla et al. (2014) both found the radio and submillimeter point source contribution to be minimal near $150 \mathrm{GHz}$. Therefore, CIB/CMB contamination in our map is expected to be small.

SZ and X-ray imaging are sensitive to the line of sight integrals of pressure (Equation (1)) and density squared, respectively; for bremsstrahlung emission, X-ray surface brightness has only a weak dependence on temperature, 
$S_{\mathrm{X}} \propto \int n_{\mathrm{e}}^{2} T_{\mathrm{e}}^{1 / 2} d \ell$. Differences between the SZ and X-ray maps ( $y$ and $S_{\mathrm{X}}$ ) can therefore be due to temperature substructure or to the differing line of sight distribution of the gas (e.g., clumping or asphericity).

The location of the SZ signal at $>6 \sigma$ broadly agrees with the location of the hottest gas found in the temperature and pseudopressure maps (Figure 3, middle and right, respectively), but we find no clear evidence for shock-heated gas at the resolution of the X-ray spectroscopy. For the high-significance $(>6 \sigma) \mathrm{SZ}$ region, we extracted spectra from both XMMNewton observations, and X-ray counts within $R_{500}$, using the same fitting procedure and plasma model as for deriving the temperature. We find only a marginal enhancement of $T_{\mathrm{X}}=11.33_{-1.61}^{+2.35} \mathrm{keV}$ over the global temperature $T_{\mathrm{X}, 500}=8.74_{-0.56}^{+0.58} \mathrm{keV}$, which agrees with that found by Planck Collaboration IV (2013).

This leaves the possibility that the SZ/X-ray offset is due to an irregular gas distribution along the line of sight or the breakdown of the assumption that pseudo-pressure and SZ features should directly match. The so-called "slab approximation," which treats the line of sight temperature as isothermal in each spectroscopic bin and assumes the path length through the cluster is a constant (e.g., Mroczkowski et al. 2012; Planck Collaboration X 2013), may not hold for complicated merger geometries.

\section{CONCLUSIONS}

We present the first high significance maps of the SZ effect with GISMO, revealing substructure in the Planck-selected cluster PLCK G147.3-16.6. The core morphology mimics the appearance of the X-ray observation (reported here and in Planck Collaboration IV 2013), but is notably offset from their $\mathrm{X}$-ray counterparts. The presence of the giant radio halo reported in van Weeren et al. (2014) further supports the hypothesis that this system is likely a merger.

This GISMO observation demonstrates that a comparable level of detail in a moderately high- $z$ cluster can now be obtained from large, ground-based telescopes in a similar amount of time as that currently required for X-ray observations. This adds to a small but growing number of instruments that have imaged the SZ effect at resolutions better than $20^{\prime \prime}$, which include such instruments as Nobeyama (e.g., Komatsu et al. 2001; Kitayama et al. 2004), MUSTANG (e.g., Mason et al. 2010), CARMA (e.g., Plagge et al. 2010), and NIKA (e.g., Adam et al. 2014, 2015). New and future observations with GISMO and GISMO-2 (Staguhn et al. 2012) will probe high- $z$ cluster mergers further, or confirm cluster candidates from SZ, X-ray, and optical surveys.

We thank all of the staff at the IRAM 30 m for their support, and Israel Hermelo in particular. We also thank Rafael Eufrásio and Alexander Karim for their input on optimizing observational strategies with GISMO+IRAM, and we thank the anonymous referee for the diligence that led to vast improvements in our analysis of the GISMO SZ data. And finally, we thank Monique Arnaud for her insightful comments on the XMM-Newton observations.

This research was performed while TM held a National Research Council Research Associateship Award at the Naval Research Laboratory (NRL). Basic research in radio astronomy at NRL by TM and TEC is supported by 6.1 Base funding. EB is supported in part by NASA ADP grant NNX13AE83G. RJvW is supported by NASA through the Einstein Postdoctoral grant number PF2-130104 awarded by the Chandra X-ray Center, which is operated by the Smithsonian Astrophysical Observatory for NASA under contract NAS8-03060. HTI is supported by the National Radio Astronomy Observatory, a facility of the National Science Foundation (NSF) operated under cooperative agreement by Associated Universities, Inc. The GISMO instrument and team are supported through NSF ATI grants 1020981 and 1106284. IRAM is supported by INSU/CNRS (France), MPG (Germany), and IGN (Spain).

\section{REFERENCES}

Adam, R., Comis, B., Macías-Pérez, J. F., et al. 2014, A\&A, 569, A66 Adam, R., Comis, B., Macías-Pérez, J.-F., et al. 2015, A\&A, 576, A12 Arnaud, M., Pratt, G. W., Piffaretti, R., et al. 2010, A\&A, 517, A10 Bleem, L. E., Stalder, B., de Haan, T., et al. 2015, ApJS, 216, 27 Böhringer, H., Voges, W., Huchra, J. P., et al. 2000, ApJS, 129, 435 Bonafede, A., Intema, H. T., Brüggen, M., et al. 2014, MNRAS, 444, L44 Brunetti, G., \& Jones, T. W. 2014, IJMPD, 23, 30007

Bulbul, E., Smith, R. K., \& Loewenstein, M. 2012, ApJ, 753, 54 Carlstrom, J. E., Ade, P. A. R., Aird, K. A., et al. 2011, PASP, 123, 903 Feretti, L., Giovannini, G., Govoni, F., \& Murgia, M. 2012, A\&A, 20, 54 Fowler, J. W., Acquaviva, V., Ade, P. A. R., et al. 2010, ApJ, 722, 1148 George, E. M., Reichardt, C. L., Aird, K. A., et al. 2015, ApJ, 799, 177 Gralla, M. B., Crichton, D., Marriage, T. A., et al. 2014, MNRAS, 445, 460 Hasselfield, M., Hilton, M., Marriage, T. A., et al. 2013, JCAP, 7, 8 Kitayama, T., Komatsu, E., Ota, N., et al. 2004, PASJ, 56, 17 Komatsu, E., Matsuo, H., Kitayama, T., et al. 2001, PASJ, 53, 57 Kovács, A. 2008, Proc. SPIE, 7020, 1

Mason, B. S., Dicker, S. R., Korngut, P. M., et al. 2010, ApJ, 716, 739 Mroczkowski, T., Dicker, S., Sayers, J., et al. 2012, ApJ, 761, 47 Plagge, T., Benson, B. A., Ade, P. A. R., et al. 2010, ApJ, 716, 1118 Planck Collaboration I 2011, A\&A, 536, A1 Planck Collaboration IV 2013, A\&A, 550, A130 Planck Collaboration VIII 2011, A\&A, 536, A8 Planck Collaboration X 2013, A\&A, 554, A140 Planck Collaboration XXIX 2014, A\&A, 571, A29 Romer, A. K., Collins, C. A., Böhringer, H., et al. 1994, Natur, 372, 75 Sanders, J. S. 2006, MNRAS, 371, 829

Sayers, J., Mroczkowski, T., Czakon, N. G., et al. 2013, ApJ, 764, 152 Staguhn, J. G., Benford, D. J., Allen, C. A., et al. 2006, Proc. SPIE, 6275, 1 Staguhn, J. G., Benford, D. J., Fixsen, D. J., et al. 2012, Proc. SPIE, 8452, 0 Staguhn, J. G., Kovács, A., Arendt, R. G., et al. 2014, ApJ, 790, 77 Sunyaev, R. A., \& Zel'dovich, Y. B. 1972, CoASP, 4, 173 van Weeren, R. J., Intema, H. T., Lal, D. V., et al. 2014, ApJL, 781, L32 Voges, W., Aschenbach, B., Boller, T., et al. 1999, A\&A, 349, 389 